\title{
Quantitative Pharmaceutical Analysis Based on the PCA of Raman Spectra
}

\author{
JUAN CARLOS MARTINEZ-ESPINOSA ${ }^{1}$, OSVALDO RODRIGUEZ-VILLALON², \\ TEODORO CORDOVA-FRAGA ${ }^{3}$, EDUARDO PEREZ-CARETA ${ }^{2}$, \\ JOSE RAFAEL GUZMAN-SEPULVEDA ${ }^{4}$, LAURA MADRID-MOLINA ${ }^{1,5}$, \\ RAFAEL GUZMAN-CABRERA ${ }^{2 *}$ \\ ${ }^{1}$ Instituto Politecnico Nacional-UPIIG, Guanajuato Puerto Interior, Silao de la Victoria, Guanajuato, MEX, 36275 \\ ${ }^{2}$ Universidad de Guanajuato, km 3.5 + 1.8 road Salamanca-Valle de Santiago, Salamanca,Guanajuato, MEX, 36730 \\ ${ }^{3}$ Universidad de Guanajuato-DCI, Loma del bosque 103 Fracc. Lomas del Campestre, Leon, \\ Guanajuato, MEX, 37150 \\ ${ }^{4}$ Centro de Investigación y de Estudios Avanzados del IPN, Unidad Monterrey. Vía del Conocimiento 201, Parque de \\ Investigación e Innovacion Tecnologica, km 9.5 de la Autopista Nueva al Aeropuerto. CP 66600. Apodaca, Nuevo Leon, \\ México \\ ${ }^{5}$ NANOBIOTECH A.C.-Innovation Park AGROBIOTEG, Av. Camino Real de Guanajuato S/N, Ejido de Juarez, Irapuato, \\ Guanajuato 36630, Mexico
}

Abstract. In the present work we examine the distribution of ciprofloxacin drug as an active ingredient in tablets samples from eight different laboratories. Additionally, control samples with different concentration were prepared for calibration. Raman spectra were recorded from all samples, including controls, and a principal component analysis (PCA) was implemented on them. The results of the PCA on control samples show that a clear discrimination among the different samples can be achieved and, more importantly, that the principal component of the Raman spectra has a welldefined, linear relation with the concentration of ciprofloxacin. Such a strong, statistically significant linear correlation obtained for the control samples constitutes our calibration for the quantitative analysis of commercial pharmaceutical compounds.

Keywords: principal components analysis, Raman spectroscopy, ciprofloxacin, pharmaceutical compounds

\section{Introduction}

Non-invasive techniques for the analysis of biological material are key to clinicians and researchers, yet there are few devices that can provide this capability. In this regard, Raman spectroscopy is well-known to be an useful tool as it can be used to measure the 'fingerprint' of chemical compositions, based on the frequency shift of the scattered light, which relates to transitions between different molecular vibration levels of the compounds in the sample $[1,2]$. Some important aspects of Raman spectroscopy are that i) it can provide information that is distinct from that generated by light microscopy of stained preparations; ii) it has the potential to avoid human influence in the interpretation of the data collected e.g., as opposed to the skilled personnel required in stain-based microscopy; and iii) it permits an efficient identification and classification of samples, thus facilitating the identification of abnormal features. These capabilities have proved useful in outstanding applications such as cancer detection [3,4] and diagnosis [5], as well as pharmaceutical analysis [6-8].

Regarding the use of Raman spectroscopy in cancer applications, extensive research has demonstrated that Raman spectroscopy measurements on biopsies can help pathologists to identify the tumor margins in a fast and precise way thus providing a valuable support to gold-standard techniques for clinical diagnostics [9-13]. More specifically, some of the recent achievements include the

$\overline{\text { *emailguzmanc@ugto.mx }}$ 
discrimination between pre-cancers and epithelial cancers; the detection of breast cancer detection in serum samples; and, the detection and monitoring of leukemia in patients under chemotherapy treatment [14].

On the other hand, ensuring correct formulation and efficient tools for processes control are critical to ensure high-quality pharmaceutical products, since small variations could result in significant changes of the properties and bioavailability of the drug, potentially affecting human health $[15,16]$. Given its importance, the quality control of pharmaceutical products includes the use of several different techniques. Most of them are primarily spectroscopy-based across the ultraviolet, visible, and infrared (IR) parts of the spectrum [17]. More specifically, the IR spectral window is of interest because that is where vibrational techniques operate $[18,19]$, which have been efficient for the characterization of solid dosage forms; for the identification of possible sources of error in manufacturing processes; for drug identification [20]; for the detection of polymorphisms and other excipients in drugs [21, 22]; as well as detecting counterfeits in a rapid, environmentally friendly, and nondestructive manner [23, 24].

Moreover, the existence of a natural bridge between the two above-mentioned fields has been demonstrated based on Raman spectroscopy studies of drug-cells interactions [25-29].

A common methodology on the Raman spectra recorded relies on the analysis of principal components (principal component analysis; PCA) and linear discriminant analysis, which has proved robust and highly sensitive for the discrimination between different biochemical compounds [30, 31], given its statistical nature to perform an eigenvalue decomposition of the descriptive data covariance.

In the present work we examine the distribution of ciprofloxacin drug as an active ingredient, by means of the PCA applied on Raman spectra measured on both commercial samples from different laboratories and calibration samples prepared with known concentrations of the drug (Sigma Aldrich: CAS 85721-33-1). Overall, as we will show throughout the paper, our approach demonstrates that Raman spectroscopy in conjunction with statistical tools such as PCA can be an efficient tool for the quantitative analysis of pharmaceutical compounds.

\section{Materials and methods \\ Sample preparation}

In this research work, fourteen pharmaceutical samples were evaluated. Eight samples containing ciprofloxacin as its active ingredient and a sample of pure excipient (in this case starch was used as an excipient medium). For samples containing the active substance, eight samples from different laboratories in the form of compressed tablets of the antibiotic ciprofloxacin were purchased from different commercial establishments. Five samples with different concentrations of ciprofloxacin as active ingredient were prepared $(20 \%, 40 \%, 60 \%, 80 \%$, and $100 \%)$. A sample of starch as an excipient was also prepared (0\%; six samples in total). The $100 \%$ stock reference solution was prepared from $1 \mathrm{~g}$ of ciprofloxacin (Sigma Aldrich CAS 85721-33-1: $\mathrm{C}_{17} \mathrm{H}_{18} \mathrm{FN}_{3} \mathrm{O}_{3}$, model weight: $331.34 \mathrm{~g}$, purity: $>=$ 98\%), and then $0.198 \mathrm{~mL}$ of a hydrochloric acid solution (Sigma Aldrich CAS 7647-01-0: HCl, molecular weight: $36.46 \mathrm{~g}$ ). Thus, obtaining the compound of ciprofloxacin hydrochloride monohydrate $\left(\mathrm{C}_{17} \mathrm{H}_{18} \mathrm{FN}_{3} \mathrm{O}_{3} \cdot \mathrm{HCl} \cdot \mathrm{H}_{2} \mathrm{O}\right)$. From the $100 \%$ stock solution, the samples with starch as excipient in percentages of $80 \%, 60 \%, 40 \%, 20 \%$, and $0 \%$, respectively, were prepared, thus generating different concentrations of ciprofloxacin hydrochloride monohydrate combined with an excipient.

\section{Integrated system for recording Raman spectra}

A Thermo Scientific DXR Raman microscopy system was used. The sample was focused with an integrated microscope through a 50X objective. The spectral recording conditions were as follows: for the eight commercial tablets from different laboratories, 25 spectra were recorded by each laboratory (matrix of 200 raw spectra). For the calibration samples, including the control and the excipient (100\% and 0\%), 75 spectra were recorded for each sample were recorded (matrix of 450 raw spectra). 
Generating a final matrix of 650 raw spectra, which were subjected to a principal component analysis. The entire spectral data set was recorded using a $780 \mathrm{~nm}$ laser excitation source, with $24 \mathrm{~mW}$ power and $50 \mathrm{~s}$ exposure time for each spectrum during its acquisition.

\section{Spectral block processing}

All spectral data processing was performed using MATLAB software (version R2018b). Raw spectra were processed by carrying baseline correction, smoothing and normalization to remove noise, sample florescence, and shot noise from cosmic rays, through a filter based on the baseline correction with asymmetric least squares smoothing algorithm [32]. Subsequently, MATLAB was used to implement the data compression technique: PCA.

\section{Results and discussions}

A spectral block was integrated into a database with spectra of all the samples analyzed, which include the spectra obtained from the pharmaceutical tablets acquired in different commercial establishments, as well as the control sample, which was acquired from Sigma Aldrich $\left(\mathrm{C}_{17} \mathrm{H}_{18} \mathrm{FN}_{3} \mathrm{O}_{3}\right.$, $331.34 \mathrm{~g} / \mathrm{mol}$, CAS 85721-33-1).

All the spectral blocks were taken to a baseline correction and a smoothing filter (Figure 1A), in order to eliminate background noise, cosmic noise, and noise caused by external sources. In this way, we ensure consistency in the conditions used to process the entire spectral database. Figure 1B shows a representative processed Raman spectrum (control and different laboratories). On the other hand, ciprofloxacin hydrochloride monohydrate presents different characteristic molecular vibrations at 638, $665,718,752,787,805,1024,1253,1273,1493,1548,1625$ and $1708 \mathrm{~cm}^{-1}$.

A.

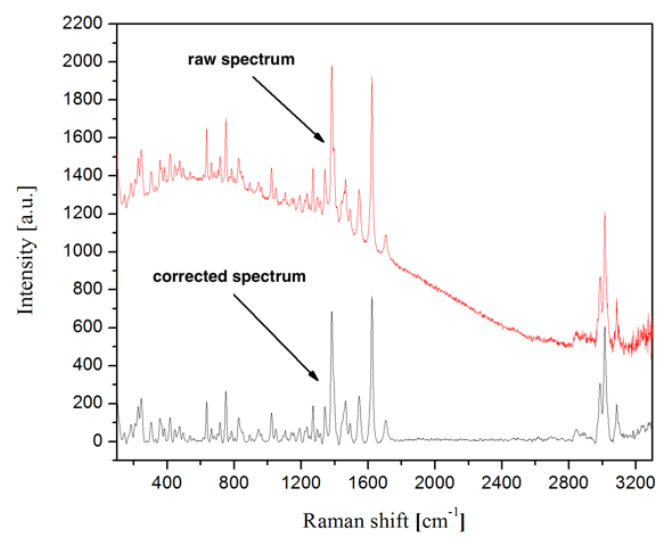

B.

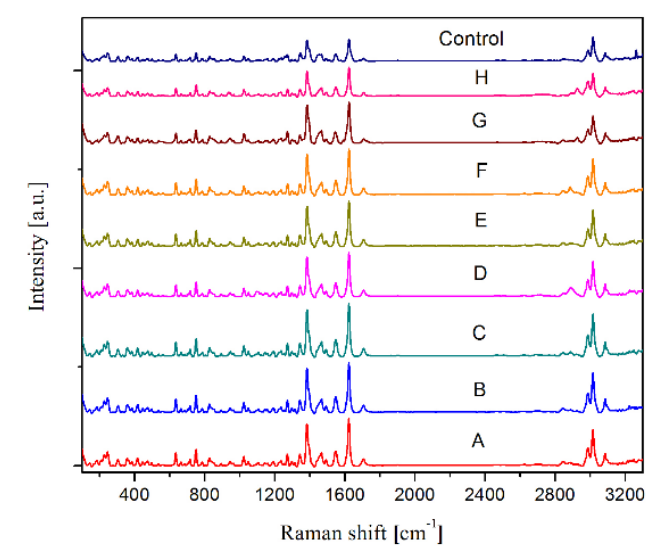

Figure 1. Raman spectral preprocessing. A. Baseline correction and smoothing: the red line corresponding to raw spectrum, and the result of the processed spectrum is indicated with the black line. B. Representative average Raman spectra of each pharmaceutical tablet (active ingredient and excipient: samples A-H) corresponding to the different laboratories analyzed. As well as the control average Raman spectrum prepared from $\mathrm{C}_{17} \mathrm{H}_{18} \mathrm{FN}_{3} \mathrm{O}_{3}$ (Sigma aldrich CAS 85721-33-1)

From the reference ciprofloxacin compound, four samples were prepared mixed with different concentrations between the active ingredient and the excipient, in addition to the pure active ingredient and one sample of pure excipient, having a total of six samples to verify the biochemical changes in function of the concentration of ciprofloxacin as an active principle. We observed that in the range of 1595 and $1650 \mathrm{~cm}^{-1}$, a molecular vibration attributed to $\mathrm{n}(\mathrm{COOH}), \mathrm{n}(\mathrm{C} 50)$ is located, in which the intensity of the vibrational band changes as a function of the concentration of the active substance (Figure 2A).

Thus, we selected the spectral window between $1595 \mathrm{~cm}^{-1}$ and $1650 \mathrm{~cm}^{-1}$ to carry out the PCA for the entire spectral block composed of 450 raw spectra ( 75 spectra per sample; in Figure 2A the error 
bars represent the standard deviation across the 75 samples). Within this range, the amplitude of the main peak, which is located at around $1624 \mathrm{~cm}^{-1}$ (attributed to $\mathrm{n}(\mathrm{COOH}), \mathrm{n}(\mathrm{C} 50)$ ), is observed to increase with the concentration of ciprofloxacin (Figure 2A). In order to verify the changes in concentrations and define a correlation function, we generated a matrix of spectra for all samples, including the pure excipient of the starch. The PCA was applied within the region from $1595 \mathrm{~cm}^{-1}$ to $1650 \mathrm{~cm}^{-1}$, as shown in Figure $2 \mathrm{~A}$, and the intensity of the peak increases linearly with the concentration of the active principle (Figure 2B).
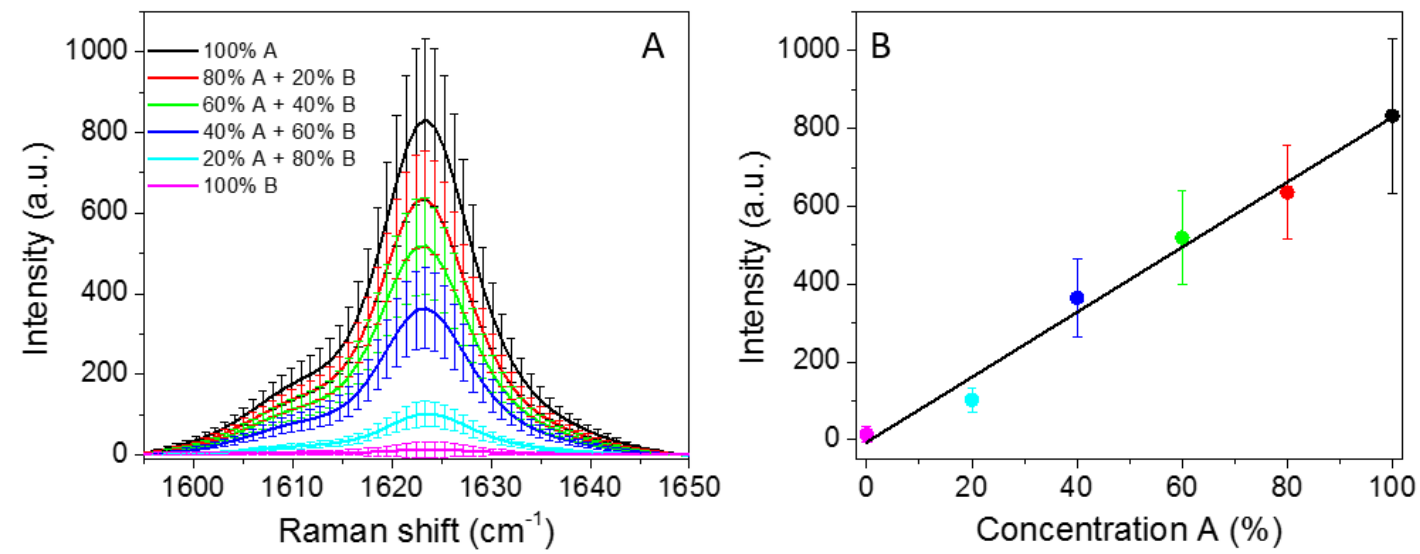

Figure 2. A. Spectra recorded in the range of 1595 to $1650 \mathrm{~cm}^{-1}$, for samples with varying concentration of ciprofloxacin, as indicated. The solid line and error bars in each curve represent the average and standard deviation, respectively, across the 75 spectra

recorded per sample (450 spectra in total). B. The peak intensity at the Raman shift of $1624 \mathrm{~cm}^{-1}$ increases linearly with the concentration of ciprofloxacin; the color of each marker in panel (b) corresponds to the color of each curve in panel (a)

In Figure 3A and 3B, we show the weight distribution for the first three principal components (PC1 versus PC2 and PC1 versus PC 3 ). The calibration curve was obtained based on the displacement of PC 1 as a function of the concentration of the active principle and the excipient.

A.

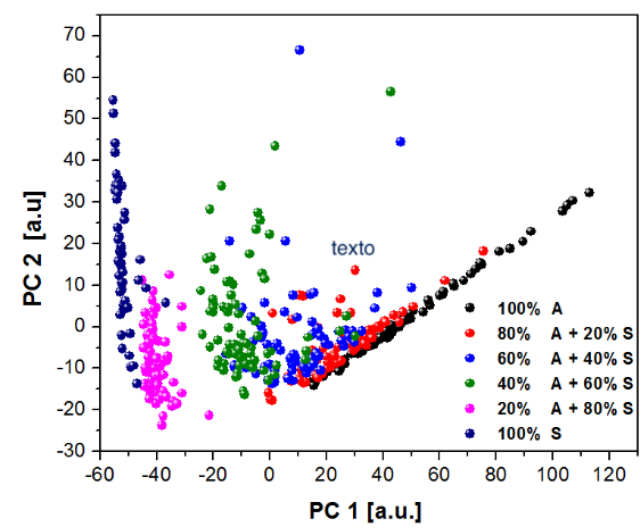

B.

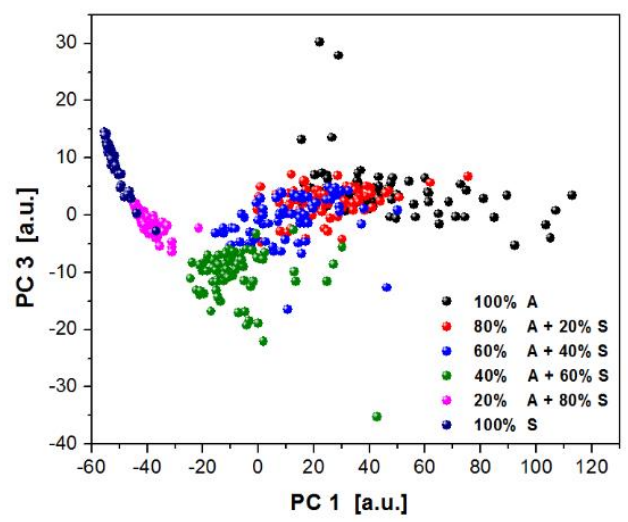

Figure 3. Results of the PCA applied to the calibration spectral database. The behavior of the weights for component PC1 shows a change depending on the concentration of the active substance. A. PC1 vs PC2 weights plotting. B. PC1 vs PC3 weights plotting

This same behavior is also observed when the first three main components (PC1, PC2 and PC3) are plotted simultaneously. Figure 4A, corresponds only to the concentrations that were used for the generation of the calibration curve. On the other hand, in Figure 4B, we can verify the behavior of the 
spectral calibration block and the spectral block of all the laboratories analyzed (a total of 450 raw spectra).
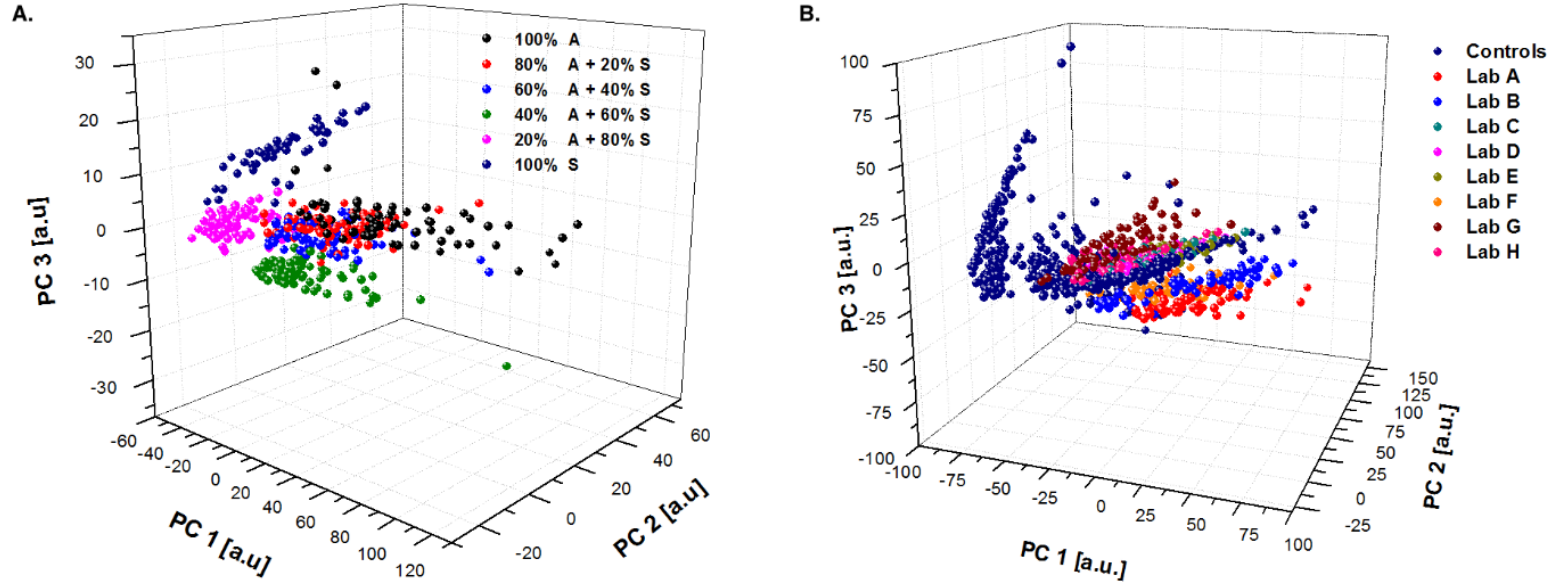

Figure 4. Comparison of plotted weight scores. A. Behavior of the weights using the first three main components. B. 3D general view of the corresponding weights for the spectral calibration block, as well as the different laboratories analyzed

The Raman spectroscopy technique is very efficient for the determination of a variation in the concentration of active substances, since these changes are reflected biochemically of any molecule under study. It is also possible to identify if an active substance is counterfeit.

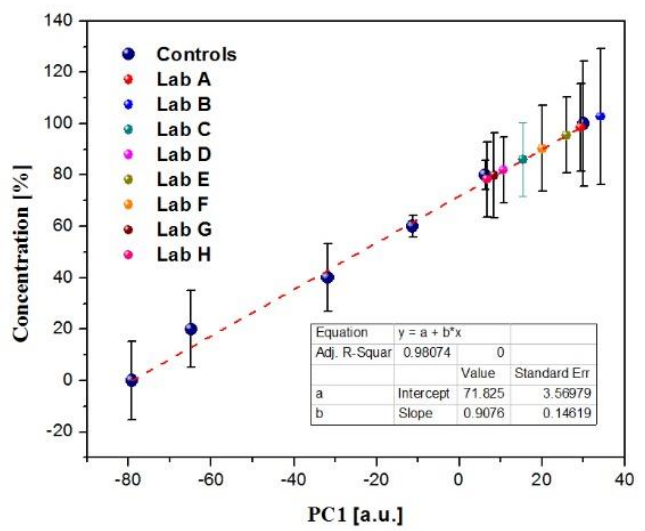

Figure 5. Evaluation of the different laboratories using the calibration curve obtained from the weights of PC1. It is observed that

the weights for the different laboratories resulted in associated concentrations greater than $80 \%$ of the active substance

From our calibration, we were able to verify that the different laboratories have $>80 \%$ of active principle in the tablets analyzed, as shown in Figure 5.

\section{Conclusions}

In this work, we demonstrate the use of Raman spectroscopy in combination with multivariate analysis as a powerful tool for pharmaceutical analysis and quantification. We worked with both control and commercial samples of ciprofloxacin; the commercial tablets were manufactured by different pharmaceutical laboratories. 
Our methodology consists on the application of PCA to the Raman spectral maps recorded, which allows measuring the mutual correlations between the different spectral groups. We processed a spectral database made up of registered spectra of the calibration samples by means of PCA; based on the first principal component (PC1), we obtained a baseline correlation with respect to the concentration of the active ingredient (Ciprifloxacin) which allows having a calibration curve for the quantitative analysis of unknown samples.

The experimental calibration correlation suggests that the quantification of the concentration in commercial ciprofloxacin tablets is directly linked to the intensity of the vibrational peak located between $1595 \mathrm{~cm}^{-1}$ and $1650 \mathrm{~cm}^{-1}$. Following this approach, we applied the PCA to the Raman spectral maps recorded for commercial ciprofloxacin tablets, and we evaluated the average value of PC1 for each laboratory analyzed (from laboratory A to $\mathrm{H}$ ). By using our calibration measurements, we were able to verify that the ciprofloxacin tablets from the different laboratories contain more than $80 \%$ of the active ingredient in the analyzed tablets (considering that $100 \%$ is for a $500 \mathrm{mg}$ preparation in a commercial tablet).

Importantly, the methodology presented is general and it can be extended to other pharmaceutical compounds, as well as to larger scale data collections e.g., to analyze samples from a larger number of sources. We consider that future work could develop a more detailed calibration correlation using high-performance liquid chromatography as an analytical method.

Finally, the quantitative assessment provided by our approach can be useful either to improve the accuracy in the dosage in cases of delicate diagnoses, or to identify adulterations in the drug formulation. Both of these goals are of critical importance to carry out quantitative measurements through alternative and high-precision spectroscopic techniques, since they can be useful to improve the quality of drug manufacturing and also identify possible adulterations or falsifications of medications that could cause side effects in people with delicate health diagnoses and who are at risk of taking drugs with altered doses.

\section{References}

1.G. VAN DALEN, E. VAN VELZEN, P. HEUSSEN, M. SOVAGO, K. VAN MALSSEN, AND J. VAN DUYNHOVEN, "Raman hyperspectral imaging and analysis of fat spreads," Journal of Raman spectroscopy, vol. 48, no. 8, pp. 1075-1084, 2017.

2.J. M. RUVALCABA-LÓPEZ et al., "Qualitative evaluation of ferritin in serum samples by Raman spectroscopy and principal component analysis," Lasers in medical science, vol. 34, no. 1, pp. 35-40, 2019.

3.P. JESS et al., "Dual beam fibre trap for Raman microspectroscopy of single cells," Optics express, vol. 14, no. 12, pp. 5779-5791, 2006.

4.L. CHEN et al., "Near-infrared confocal micro-Raman spectroscopy combined with PCA-LDA multivariate analysis for detection of esophageal cancer," Laser Physics, vol. 23, no. 6, p. 065601, 2013.

5.W. WANG, J. ZHAO, M. SHORT, AND H. ZENG, "Real-time in vivo cancer diagnosis using raman spectroscopy," Journal of biophotonics, vol. 8, no. 7, pp. 527-545, 2015.

6.T. VANKEIRSBILCK et al., "Applications of Raman spectroscopy in pharmaceutical analysis," TrAC trends in analytical chemistry, vol. 21, no. 12, pp. 869-877, 2002.

7.T. LIPIÄINEN, S. J. FRASER-MILLER, K. C. GORDON, AND C. J. STRACHAN, "Direct comparison of low-and mid-frequency Raman spectroscopy for quantitative solid-state pharmaceutical analysis," Journal of pharmaceutical and biomedical analysis, vol. 149, pp. 343-350, 2018.

8.J. MARTINEZ, J. GUZMÁN-SEPÚlVEDA, G. B. EVIA, T. CÓRDOVA, AND R. GUZMÁNCABRERA, "Enhanced Quality Control in Pharmaceutical Applications by Combining Raman Spectroscopy and Machine Learning Techniques," International Journal of Thermophysics, vol. 39, no. 6, p. 79, 2018. 
9.M. S. BERGHOLT et al., "Fiberoptic confocal raman spectroscopy for real-time in vivo diagnosis of dysplasia in Barrett's esophagus," Gastroenterology, vol. 146, no. 1, pp. 27-32, 2014.

10.I. TALEB et al., "Diagnosis of hepatocellular carcinoma in cirrhotic patients: a proof-of-concept study using serum micro-Raman spectroscopy," Analyst, vol. 138, no. 14, pp. 4006-4014, 2013.

11.S. BOREL et al., "Discrimination of normal and malignant mouse ovarian surface epithelial cells in vitro using Raman microspectroscopy," Analytical Methods, vol. 7, no. 22, pp. 9520-9528, 2015.

12.O. OLD et al., "Vibrational spectroscopy for cancer diagnostics," Analytical Methods, vol. 6, no. 12, pp. 3901-3917, 2014.

13.A. SAHU et al., "Raman spectroscopy and cytopathology of oral exfoliated cells for oral cancer diagnosis," Analytical Methods, vol. 7, no. 18, pp. 7548-7559, 2015.

14.L. P. MOREIRA, L. SILVEIRA JR, M. T. T. PACHECO, A. G. DA SILVA, AND D. D. F. M. ROCCO, "Detecting urine metabolites related to training performance in swimming athletes by means of Raman spectroscopy and principal component analysis," Journal of Photochemistry and Photobiology B: Biology, vol. 185, pp. 223-234, 2018.

15.S. NEUBERGER AND C. NEUSUß, "Determination of counterfeit medicines by Raman spectroscopy: Systematic study based on a large set of model tablets," Journal of pharmaceutical and biomedical analysis, vol. 112, pp. 70-78, 2015.

16.K. DÉGARDIN, Y. ROGGO, F. BEEN, AND P. MARGOT, "Detection and chemical profiling of medicine counterfeits by Raman spectroscopy and chemometrics," Analytica chimica acta, vol. 705, no. 1-2, pp. 334-341, 2011.

17.C. Y. BASTIDAS, C. VON PLESSING, J. TRONCOSO, AND R. D. P. CASTILLO, "Evaluation of the microscopic distribution of florfenicol in feed pellets for salmon by Fourier Transform infrared imaging and multivariate analysis," Journal of pharmaceutical and biomedical analysis, vol. 152, pp. 257-263, 2018.

18.K. DÉGARDIN, A. GUILlEMAIN, N. V. GUERREIRO, AND Y. ROGGO, "Near infrared spectroscopy for counterfeit detection using a large database of pharmaceutical tablets," Journal of pharmaceutical and biomedical analysis, vol. 128, pp. 89-97, 2016.

19.Y. V. KAZAKEVICH AND R. LOBRUTTO, HPLC for pharmaceutical scientists. John Wiley \& Sons, 2007.

20.A. DE JUAN, R. TAULER, R. DYSON, C. MARCOLli, M. RAULT, AND M. MAEDER, "Spectroscopic imaging and chemometrics: a powerful combination for global and local sample analysis," TrAC Trends in Analytical Chemistry, vol. 23, no. 1, pp. 70-79, 2004.

21.S. C. BROWN, M. CLAYBOURN, D. SIEVWRIGHT, V. FEARNSIDE, AND C. ASHMAN, "Lean Raman imaging for rapid assessment of homogeneity in pharmaceutical formulations," Applied spectroscopy, vol. 64, no. 4, pp. 442-447, 2010.

22.T. KOIDE, Y. YAMAMOTO, T. FUKAMI, N. KATORI, H. OKUDA, AND Y. HIYAMA, "Analysis of distribution of ingredients in commercially available clarithromycin tablets using nearinfrared chemical imaging with principal component analysis and partial least squares," Chemical and Pharmaceutical Bulletin, vol. 63, no. 9, pp. 663-668, 2015.

23.S. LOHUMI, M. S. KIM, J. QIN, AND B.-K. CHO, "Raman imaging from microscopy to macroscopy: Quality and safety control of biological materials," TrAC Trends in Analytical Chemistry, vol. 93, pp. 183-198, 2017.

24.L. M. KANDPAL, B.-K. CHO, J. TEWARI, AND N. GOPINATHAN, "Raman spectral imaging technique for API detection in pharmaceutical microtablets," Sensors and Actuators B: Chemical, vol. 260, pp. 213-222, 2018.

25.S. ŚŠ́Ć, "Chemical imaging of pharmaceutical granules by Raman global illumination and nearinfrared mapping platforms," Analytica chimica acta, vol. 611, no. 1, pp. 73-79, 2008.

26.W. H. DOUB, W. P. ADAMS, J. A. SPENCER, L. F. BUHSE, M. P. NELSON, AND P. J. TREADO, "Raman chemical imaging for ingredient-specific particle size characterization of aqueous 
suspension nasal spray formulations: a progress report," Pharmaceutical research, vol. 24, no. 5, pp. 934-945, 2007.

27.J. LING, S. D. WEITMAN, M. A. MILLER, R. V. MOORE, AND A. C. BOVIK, "Direct Raman Imaging Techniques for Studying the Subcellular Distribution of a Drug."

28.M. B. MAMIAN-LOPEZ AND R. J. POPPI, "SERS hyperspectral imaging assisted by MCR-ALS for studying polymeric microfilms loaded with paracetamol," Microchemical Journal, vol. 123, pp. 243-251, 2015.

29.Y. ROGGO, K. DEGARDIN, AND P. MARGOT, "Identification of pharmaceutical tablets by Raman spectroscopy and chemometrics," Talanta, vol. 81, no. 3, pp. 988-995, 2010.

30.G. S. BUMBRAH AND R. M. SHARMA, "Raman spectroscopy-Basic principle, instrumentation and selected applications for the characterization of drugs of abuse," Egyptian Journal of Forensic Sciences, vol. 6, no. 3, pp. 209-215, 2016.

31.M. EDINGER, D. BAR-SHALOM, J. RANTANEN, AND N. GENINA, "Visualization and nondestructive quantification of inkjet-printed pharmaceuticals on different substrates using Raman spectroscopy and Raman chemical imaging," Pharmaceutical research, vol. 34, no. 5, pp. 1023-1036, 2017.

32.A. M. MARTÍNEZ AND A. C. KAK, "Pca versus lda," IEEE transactions on pattern analysis and machine intelligence, vol. 23, no. 2, pp. 228-233, 2001

$\overline{\text { Manuscript received: } 17.12 .2019}$ 